TITLE:

\title{
Homogeneous and elongation-free 3D microfabrication by a femtosecond laser pulse and hologram
}

\section{$\operatorname{AUTHOR(S):~}$}

Yamaji, M.; Kawashima, H.; Suzuki, J.; Tanaka, S.; Shimizu, M.; Hirao, K.; Shimotsuma, Y.; Miura, K.

\section{CITATION:}

Yamaji, M....[et al]. Homogeneous and elongation-free 3D microfabrication by a femtosecond laser pulse and hologram. JOURNAL OF APPLIED PHYSICS 2012, 111(8): 083107.

\section{ISSUE DATE:}

2012-04-15

URL:

http://hdl.handle.net/2433/160651

\section{RIGHT:}

Copyright 2012 American Institute of Physics. This article may be downloaded for personal use only. Any other use requires prior permission of the author and the American Institute of Physics. The following article appeared in JOURNAL OF APPLIED PHYSICS 111, 083107 (2012) and may be found at http://link.aip.org/link/?jap/111/083107 


\section{AIP $\mid$ Appolied Physics}

\section{Homogeneous and elongation-free 3D microfabrication by a femtosecond laser pulse and hologram}

M. Yamaji, H. Kawashima, J. Suzuki, S. Tanaka, M. Shimizu et al.

Citation: J. Appl. Phys. 111, 083107 (2012); doi: 10.1063/1.4705286

View online: http://dx.doi.org/10.1063/1.4705286

View Table of Contents: http://jap.aip.org/resource/1/JAPIAU/v111/i8

Published by the American Institute of Physics.

\section{Related Articles}

Low-frequency Raman scattering from Si/Ge nanocrystals in different matrixes caused by acoustic phonon quantization

J. Appl. Phys. 112, 054318 (2012)

Copper oxide decorated multi-walled carbon nanotubes/ferroelectric liquid crystal composites for faster display devices

J. Appl. Phys. 112, 054309 (2012)

Permeability and magnetic properties of $\mathrm{Al} 3+$ substituted Ni0.7Zn0.3Fe2O4 nanoparticles

J. Appl. Phys. 112, 053908 (2012)

Nanoparticle formation in a cavitation bubble after pulsed laser ablation in liquid studied with high time resolution small angle x-ray scattering

Appl. Phys. Lett. 101, 103104 (2012)

Metal nanoantenna plasmon resonance lineshape modification by semiconductor surface native oxide J. Appl. Phys. 112, 044315 (2012)

\section{Additional information on J. Appl. Phys.}

Journal Homepage: http://jap.aip.org/

Journal Information: http://jap.aip.org/about/about_the_journal

Top downloads: http://jap.aip.org/features/most_downloaded

Information for Authors: http://jap.aip.org/authors

\section{ADVERTISEMENT}

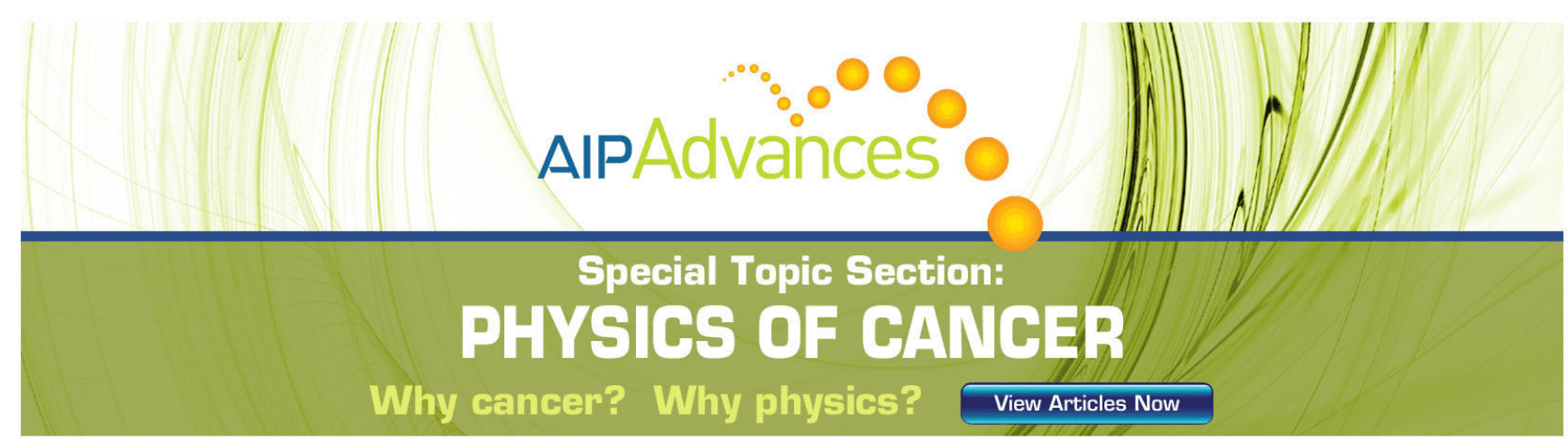




\title{
Homogeneous and elongation-free 3D microfabrication by a femtosecond laser pulse and hologram
}

\author{
M. Yamaji, ${ }^{1, a)}$ H. Kawashima, ${ }^{2}$ J. Suzuki, ${ }^{2}$ S. Tanaka, ${ }^{2}$ M. Shimizu, ${ }^{1}$ K. Hirao, ${ }^{1}$ \\ Y. Shimotsuma, ${ }^{1}$ and K. Miura ${ }^{1}$ \\ ${ }^{1}$ Department of Material Chemistry, Graduate School, Kyoto University, Kyoto 615-8510, Japan \\ ${ }^{2}$ Nanoglass Research Center, New Glass Forum, Ibaraki 300-2635, Japan
}

(Received 6 October 2011; accepted 16 March 2012; published online 23 April 2012)

\begin{abstract}
A new 3D microfabrication method has been developed, which uses only a single femtosecond laser pulse and a hologram. For the microfabrication inside transparent materials, the optical axial elongation of the fabricated structure is a major problem that has thus far limited design flexibility, especially for the direction along the optical axis. By controlling the light intensity distribution profile and using the adequate focal length of the hologram, this problem was solved and homogeneous and elongation-free 3D microfabrication was achieved. (C) 2012 American Institute of Physics. [http://dx.doi.org/10.1063/1.4705286]
\end{abstract}

\section{INTRODUCTION}

Three dimensional microfabrication inside transparent materials by laser irradiation has achieved drastic development thus far. Various optical devices such as waveguides, ${ }^{1,2}$ couplers, ${ }^{3,4}$ gratings, ${ }^{5-7} 3 \mathrm{D}$ channels,${ }^{8-10}$ and optical data storage $^{11,12}$ have been fabricated by this method.

To create a localized structural change, an ultrashort pulsed laser with a longer wavelength than the absorption edge should be focused inside the material. The high intensity pulse induces nonlinear optical absorption processes, namely the multiphoton absorption and the avalanche ionization. ${ }^{13-17}$ These processes yield free electrons which transfer their excess energy to the lattice, causing permanent localized structural change. Compared with their longer pulse counterparts, femtosecond pulse lasers can produce higher spatial resolution microstructures because of very small heat diffusion effects.

For 3D microfabrication, the sequential laser direct writing method ${ }^{15-17}$ has thus far been standard. This method is very simple: it uses tightly focused laser pulses and only relies on controlling a triaxial sample stage with high accuracy to fabricate dots one by one. This takes prolonged fabrication time that is unrealistic for bulk production. Hence, we developed a new 3D microfabrication technique that is achieved by only a single laser pulse and a hologram. ${ }^{18,19} \mathrm{In}$ this technique, the phase distribution of the pulse is controlled by a transmissive glass hologram pattern resulting in regions where constructive interference yields a high light intensity beyond the fabrication threshold. In other words, an arbitrary 3D microstructure can be fabricated inside a transparent material without any sample stage control. This 3D microfabrication technique, therefore, has advantages such as high flexibility in designing the 3D microstructure, high accuracy in the relative position independent of that of the triaxial scanning stage, high-speed fabrication, and flexible aspect ratio of each fabricated dot independent of the numer-

${ }^{a)}$ E-mail: misty540@1999.jukuin.keio.ac.jp. ical aperture (NA) value of the objective lens. The usability of this technique is also demonstrated by several groups that use a reflective spatial light modulators (SLMs) ${ }^{20}$ instead of a transmissive glass hologram. ${ }^{21,22}$ Each of them has different advantages; SLMs can change hologram patterns sequentially, but glass holograms are applicable for higher peak power lasers, and have higher resolution than SLMs.

The biggest problem with this technique is an elongation of the fabricated structure along the optical axis. ${ }^{15}$ This difficulty is attributed to the fact that high light intensity regions are formed by the diffraction of waves which arrive with a narrow convergence angle. This is especially problematic in the case of forming closely spaced multiple dots at different depths, because the elongation length limits the minimum interlayer distance. This is the main reason why this technique has been applied to only simple microstructures. ${ }^{23-25}$

In this paper, a method to achieve homogeneous and elongation-free microfabrication is discussed. By using this technique, we successfully fabricated a V-shaped line structure oriented vertically along the optical axis.

\section{EXPERIMENTAL DETAILS}

A schematic diagram of the experimental setup used for this holographic 3D microfabrication technique is shown in Fig. 1. An amplified Ti:Sapphire femtosecond laser (Femtolasers/FEMTOPOWER compact PRO) with $800 \mathrm{~nm}$ wavelength and $8 \mathrm{~mm}$ diameter was used as the light source. The pulse duration and pulse energy were variable in the range of 26-735 fs and 10-700 $\mu \mathrm{J} /$ pulse, respectively. The repetition rate was variable in the range of $1-1000 \mathrm{~Hz}$, which is too low to cause thermal accumulation effects.

The femtosecond laser pulse penetrates through a phase distribution pattern etched onto a 1-mm-thick optically polished silica glass plate (Shin-Etsu Chemical Co., VIOSIL). A typical hologram pattern is also shown in Fig. 1. This hologram pattern images a spirally arranged series of 24 dots (left below in Fig. 1). The Fresnel-Kirchhoff diffractive integral method is used to calculate the hologram pattern. The shape of the hologram is a circle with an $8 \mathrm{~mm}$ diameter, 


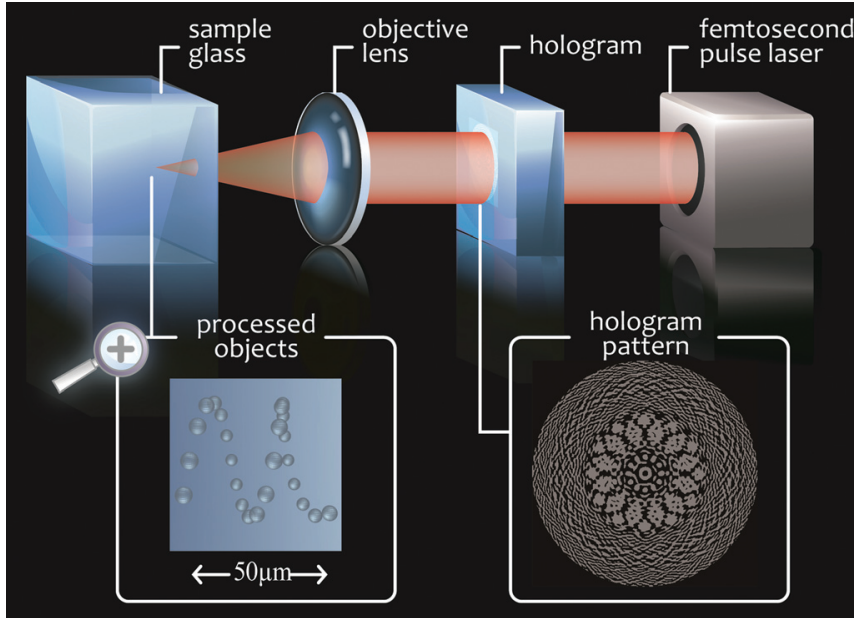

FIG. 1. A schematic diagram of the holographic 3D microfabrication system: a pulse emitted from the femtosecond pulse laser penetrates through a hologram and an objective lens and fabricates a 3D microstructure inside a sample material. The hologram pattern shown here is used for fabricating the spiral dot array in Fig. 5.

which is same as that of the femtosecond laser pulse at $1 / \mathrm{e}^{2}$ intensity in order to use as much of the incident pulse energy as possible. The holograms used in this report are binary phase holograms calculated by the optimal-rotation-angle method. ${ }^{26}$ This means that the phase distribution is controlled by the difference in the thickness between etched and non-etched regions on the glass plate. The etched depth is equal to $870 \mathrm{~nm}$, corresponding to a $\pi$ phase shift. The white pixels in the hologram pattern represent an area etched by electron beam lithography and reactive ion etching. The sidewall roughness of the step is less than $50 \mathrm{~nm}$, which is small enough to prevent the diffractive efficiency from significantly degrading. ${ }^{2}$

The reconstructed 3D micropatterns were focused by a $20 \times$ objective lens $(\mathrm{NA}=0.46)$ inside an optically polished fused silica glass sample (ES: Tosoh Quartz Corp.) in order to reach sufficiently high photon density for multi-photon absorption.

\section{RESULTS AND DISCUSSION}

\section{A. Intensity peak profile control}

We prepared an intensity variation hologram that had seven dots of different intensities to validate the relationship between the intensity distribution profile and the fabrication size. Figure 2(a) shows the schematic design of this hologram. Dots $\mathrm{A}$ to $\mathrm{F}$ have different intensity peak profiles, which are confirmed by the regenerated intensities calculated from this hologram (Fig. 2(b)).

Figure 2(c) is a scanning electron microscope (SEM) image of the microstructure produced by this hologram. It was fabricated on the surface of the silica glass, since it is difficult to quantify the diameter of the dots fabricated inside a transparent material. Seven dots of different diameters were fabricated simultaneously, demonstrating that the hologram can control multiple intensity peak profiles individually. Because intensity profiles vary sensitively with position, intensity control by the hologram is useful to achieve homogeneous microfabrication. This ablation test was done with a single laser pulse in order to avoid the integrated effect of the pulse train. Since the shape of each dot deviated from the ideal circular shape, which is often the case for the ablation of silica glass by a small number of pulses, the average diameter of 10 samples was used for the measured value.

The difference in diameter seen in Fig. 2(c) is directly attributed to the difference in the light intensity peak width. Figure 2(d) shows the relationship between the measured diameter and the calculated light intensity peak width. The width at the dashed line in Fig. 2(b) is assumed to be a fabrication condition, because the pulse fluence was set just above the threshold of a 50\% intensity dot. This result shows that the diameter becomes larger as the peak widens, and it can be said that the fabrication diameter can be controlled by the intensity distribution profile. However, significant variation is seen between these calculated and measured values. This difference is equal to the distance between the plots and the dashed line in Fig. 2(d). It is attributed to multiphoton absorption occurring as the trigger of structural change at the area where the photon density is higher than the threshold. This threshold is that of multiphoton absorption and is different from that of localized structural change or plasma formation. In fact, the volumes of the multiphoton absorption, plasma formation, and structurally changed regions are different, which was confirmed by pump-probe measurement of plasma inside silica glass. ${ }^{27}$ Multiphoton absorption is followed by avalanche ionization, electron-phonon interaction, heat diffusion, ${ }^{28}$ and shock wave expansion. ${ }^{29}$ The fabrication size is determined by all these processes, as well as material properties such as melting temperature, glass-transition point, composition, viscosity, and heat conduction coefficient. Therefore, the peak width indicates the area where enough free electrons for localized structural change are yielded, but other subsequent phenomena and factors decide the difference between this area and the actual localized structurally changed area. Heat diffusion and shock wave expansion also depend on the pulse fluence (photon density), which explains why the distance between the plot and the dashed line becomes large as the intensity peak height increases. It is arguable whether electron diffusion expands the localized structurally changed area. Although it is thought to be unable to contribute because the excess energy of the plasma is transferred to the lattice before diffusion and recombination occur, ${ }^{17,27}$ the calculation of the electron number density rate equation, which includes electron reduction via diffusion and recombination, shows good agreement with earlier studies. ${ }^{30}$

Because the factors influencing the difference between the plots and the dashed line in Fig. 2(d) are difficult to control, varying the intensity peak width is an adequate approach to control of the localized structurally changed area.

\section{B. Elongation control}

Another two holograms were prepared that produce only one dot at $100 \mathrm{~mm}$ or $500 \mathrm{~mm}$. The calculated light intensity 


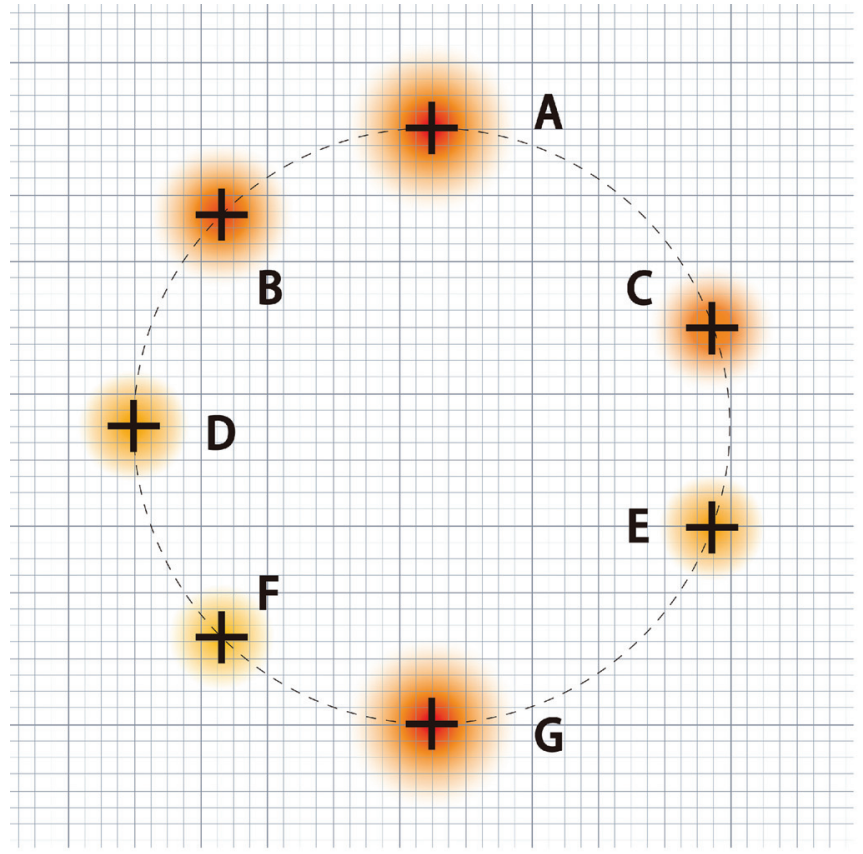

(a)

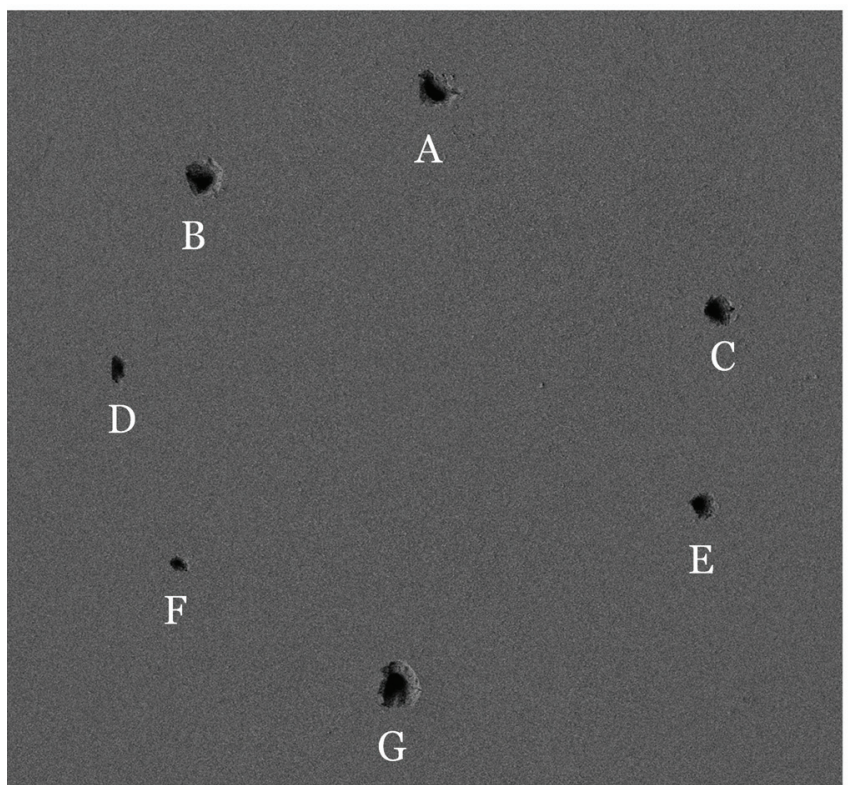

(c)

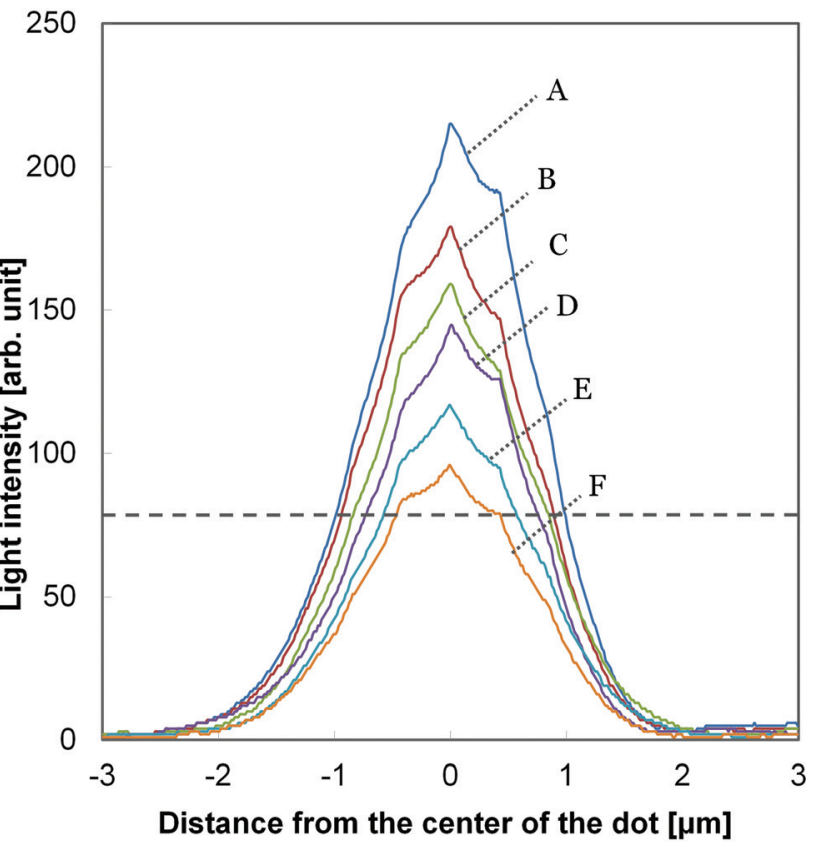

(b)

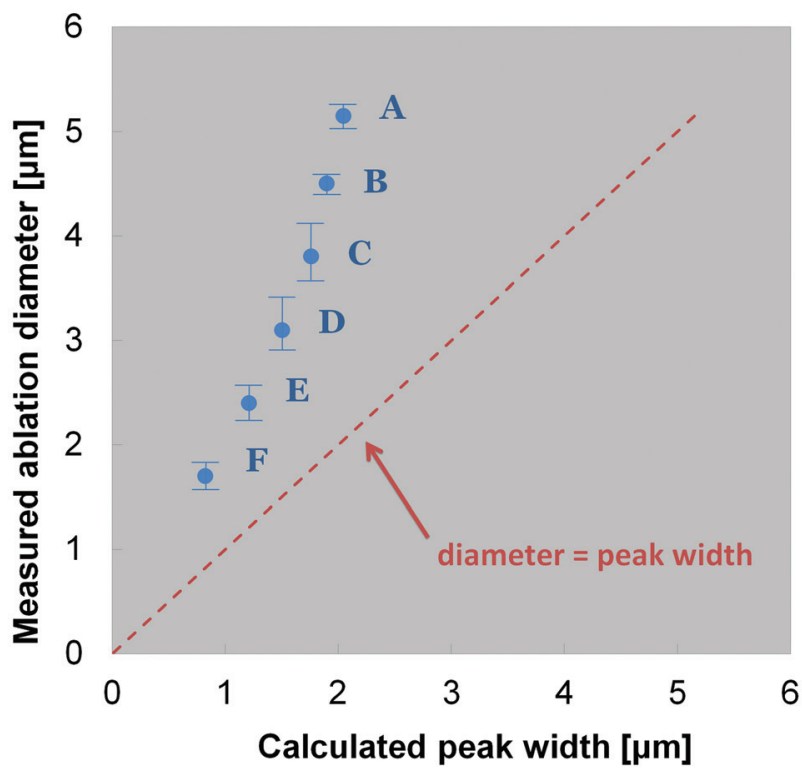

(d)

FIG. 2. (a) The design of the intensity variation hologram. The percentage indicates the maximum intensity of each dot. (b) The intensity distribution as a function of the distance from the center of the dot. (c) An SEM image of the fabricated structure using this intensity variation hologram. (d) The relationship between the average diameters of each dot and the intensity peak width. The dashed line indicates the condition in which the measured diameter and the calculated peak width are equal.

distributions of these two holograms are shown in 3D in Fig. 3(a). In this calculation, a Gaussian continuous beam is used for the input value and is assumed to penetrate through the M20 $(\mathrm{NA}=0.46)$ objective lens after the hologram. The distance between the objective lens and the hologram is adjusted so that the images of these two holograms are focused on at the same distance from the objective lens. Figure 3(b) shows the cross sectional view of Fig. 3(a) along the optical axis. These two intensity distributions show quite different profiles despite these dots being focused at the same distance from the objective lens. The full width at half maximum (FWHM) of the short $f_{H}$ (focal length of the hologram) is about seven times narrower than that of the long $f_{H}$ hologram. This means that the aspect ratio of the fabrication, which is fixed by the NA value without the hologram, varies with the hologram. Considering the fact that the objective lens controls the aspect ratio by restricting the working distance, this is one of the advantages of this $3 \mathrm{D}$ microfabrication technique. Moreover, the slope of the narrow distribution is steep, which means that fluence variation does not 


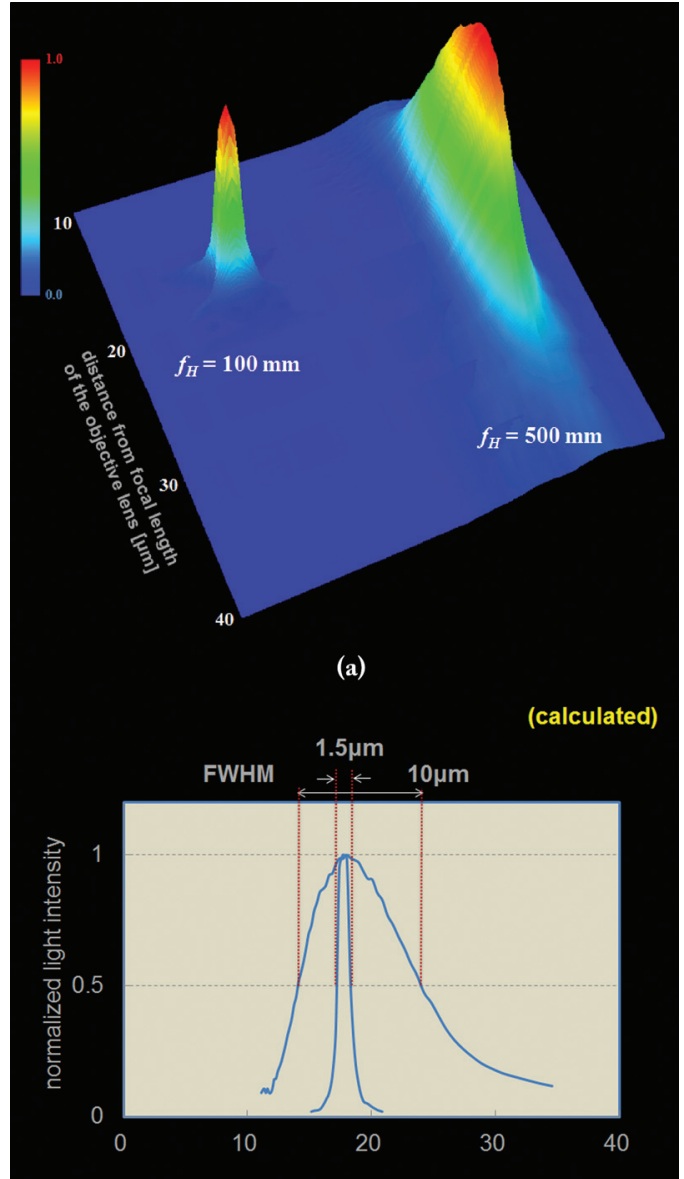

distance from focal length of the objective lens $[\mu \mathrm{m}]$

(b)

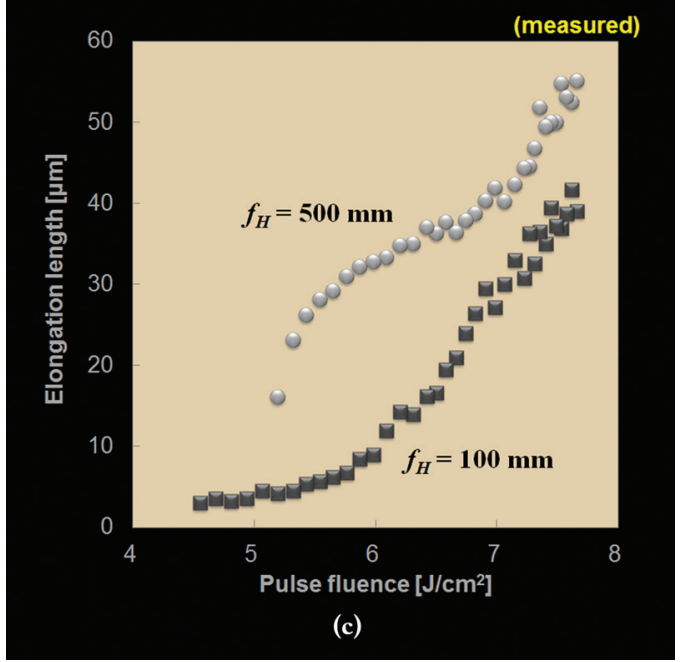

FIG. 3. (a) The regenerated light intensity distributions of two different holograms are shown as a 3D surface map. (b) The cross sectional view of Fig. 3(a) along the optical axis. (c) The measured elongation length as a function of the pulse fluence.

affect the elongation length very much. This allows easier elongation control and reduces the prolonged calculation time for adjusting peak profile exactly.

The elongation length of the dots fabricated by using these two holograms is plotted in Fig. 3(d) as a function of the pulse fluence. The difference is pronounced in the lowfluence region: the result of the short $f_{H}$ hologram shows lit- tle dependency on the fluence, whereas that of long $f_{H}$ hologram shows a steep decrease. Such abrupt change near the threshold means that precise elongation control by the pulse fluence in this region is difficult. Actually, to fabricate a dot of sub-10- $\mu \mathrm{m}$ elongation with the long $f_{H}$ hologram, the pulse fluence must have an accuracy of $0.1 \mathrm{~J} / \mathrm{cm}^{2}$, compared to $1 \mathrm{~J} / \mathrm{cm}^{2}$ with the short $f_{H}$ hologram. Fluence becomes even more critical when multiple dots are fabricated by one shot: a slight difference in intensity peak height yields a certain amount of variance in the elongation when the pulse fluence is set just above the threshold. Besides this fact, the intensity at each element has a complicated dependency especially in the direction of optical axis, which is caused mainly by the following three reasons. First is light attenuation. Though the laser pulse is a plane wave and has an extremely low beam spread angle, it turns into numerous spherical waves after passing through the hologram, which then attenuate in proportion to the distance from the hologram. This means that the dots farther from the hologram have a lower light intensity, and the curve shown in Fig. 3(c) shifts to the left. This is a great disadvantage for homogeneous microfabrication when the long $f_{H}$ hologram is used because of the abrupt decrease in elongation length near the threshold. The second reason is the focusing property of the objective lens. The light intensity at the fabrication point depends on the numerical aperture value and the distance from the focal point of the objective lens. The third reason is chromatic and spherical aberrations. If the microstructure is spread over a wide area along the optical axis, the elongation length lengthens as the depth from the sample glass surface increases. The inhomogeneity of the light intensity caused by these factors and the abrupt decrease in elongation length near the threshold make homogeneous and elongation-free microfabrications difficult when the long $f_{H}$ hologram is used.

The difference in behavior between the two series in Fig. 3(c) comes from the difference in the intensity distribution profile. By rotating Fig. 3(c) $90^{\circ}$ to the right, we can see it is similar to Fig. 3(b). If the pulse fluence is set just above the threshold, the peak width near the threshold is the area where enough free electrons for localized structural change are yielded, and as the pulse fluence increases, this area grows as the threshold intersects a wider part of the intensity peak. Considering that the factors that expand the modified region from the intensity peak width affect it almost isotropically, the elongation length along the optical axis depends on this light intensity width. This means that the hologram can control the elongation length.

Unlike the case in Fig. 2, the elongation along the optical axis suffers from the effects of defocusing and selffocusing. These effects depend on the pulse fluence, and are also the reason for the difference in the measured elongation length and the calculated intensity peak width.

\section{Avoiding duplication}

Generally, holograms project two different images, the real and virtual images. Combining the hologram and the 


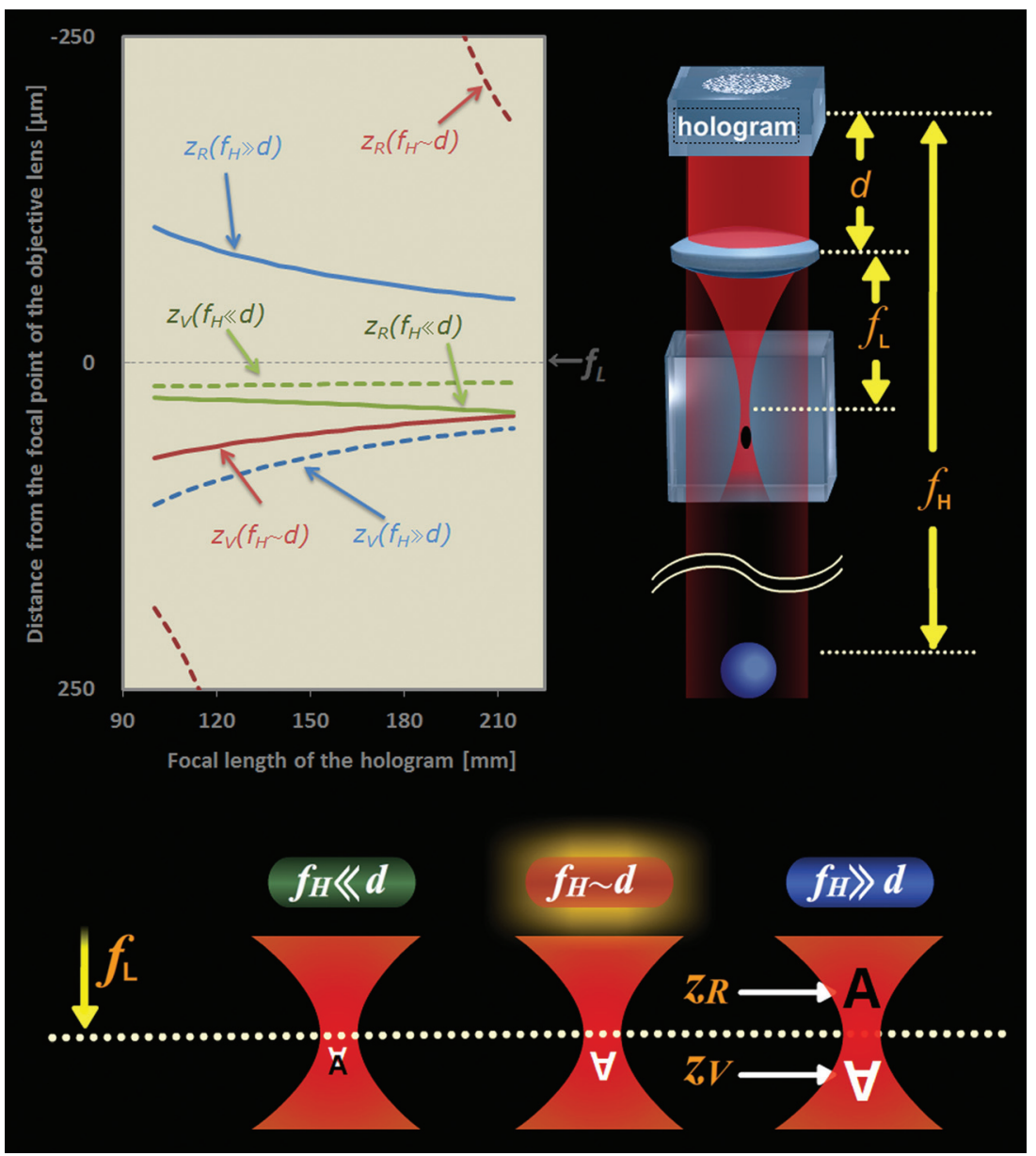

FIG. 4. The behavior of the virtual and real images is shown. The focal length of the objective lens $f_{L}$ is located at the center of the vertical axis. In the case of $f_{H} \ll d$ (green series), the virtual and real images are located at the same side of the $f_{L}$, whereas they are located opposite side in the case of $f_{H} \ll d$ (blue series). If $f_{H}$ is comparable to $d$ (red series), the real image moves toward infinite, and only the virtual image contributes to the fabrication.

objective lens focuses on the real and virtual images at the distances,

$$
\begin{aligned}
& z_{r}=f_{H} \frac{f_{L}+d}{f_{L}+d-f_{H}}, \\
& z_{v}=f_{H} \frac{f_{L}-d}{f_{L}-d+f_{H}},
\end{aligned}
$$

from the objective lens, respectively. ${ }^{31}$ Here, $d$ means the distance between the hologram and the objective lens, and $f_{L}$ means the focal length of the objective lens. If the hologram is placed before the objective lens, $z_{r}$ (solid line) and $z_{v}$ (dashed line) behave as shown in Fig. 4 as a function of the focal length of the hologram $f_{H}$. In this figure, the value far from the objective lens is plotted at the bottom side. The origin of the vertical axis $\mathrm{z}=0$ is set to the focal length of the objective lens $f_{L}$. Parameters $d$ and $f_{H}$ are $100 \mathrm{~mm}$ and 100-215 mm, respectively, which are used for the fabrication in Fig. 5. Three cases are shown in the figure: (i) $f_{H}, \ll d$, (ii) $f_{H} \gg d$, and (iii) $f_{H} \sim d$. The bottom of Fig. 4 shows the schematic diagram of these three cases. In the first case, the real and virtual images are located closely, and it is difficult to avoid the "duplication" of the fabricated structure. Although these images can be separated by increasing $f_{H}$, this also requires large $d$ value, which reduces the diffraction efficiency of the hologram. In the second case, two images are focused at opposite sides of the focal point of the objective lens. This condition allows only the virtual image to be fabricated if the glass sample positioned such that the virtual image is focused inside the sample and the real image focuses outside the sample. In the third case, the real image moves towards infinity as $d$ and $f_{H}$ become closer. It means that only the virtual image is focused inside the fabrication area without the alignment restriction of case (ii). Therefore, this case is the most appropriate for this fabrication technique. All these cases are based on the assumption that the hologram is located before the objective lens. With this arrangement, the real image is more sensitive than the virtual

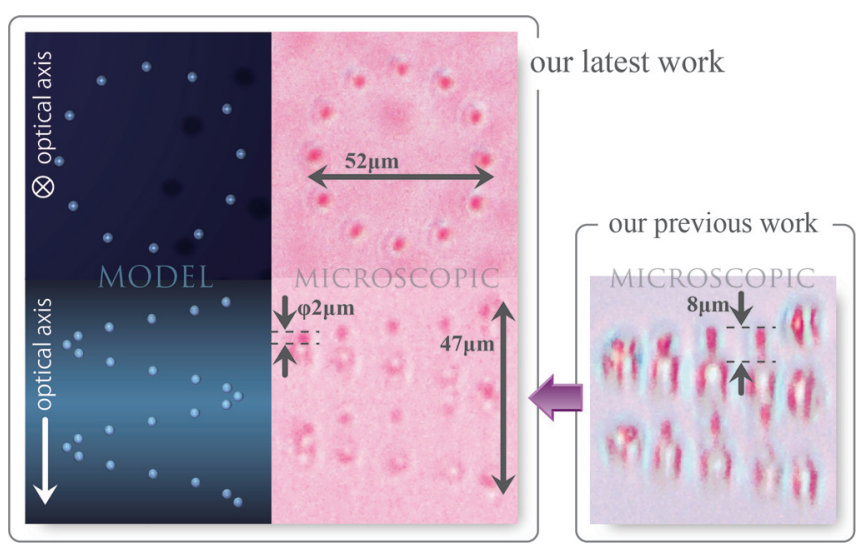

FIG. 5. The model image (left) and optical micrograph (right) of an elongation-free spiral dot array fabricated inside the silica glass is shown and compared with our previous work; (upper images) top view; (lower images) side view. 

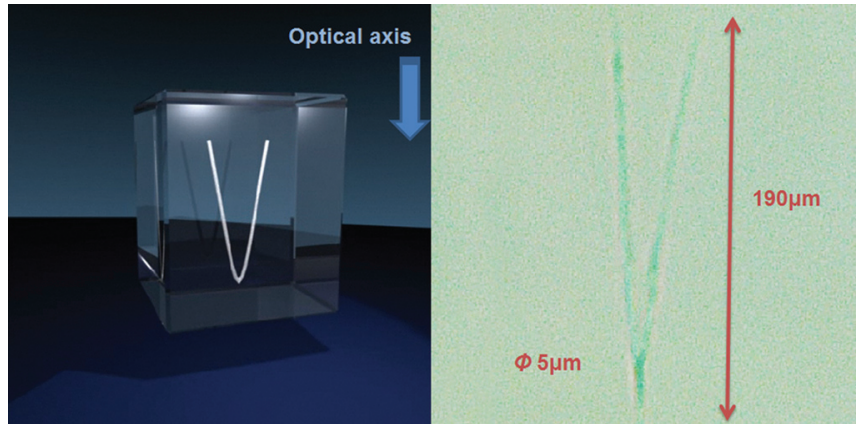

FIG. 6. The model 3D image (left) and optical micrograph (right) of an elongation-free $\mathrm{V}$-shaped line microstructure fabricated inside silica glass.

image, and easier to remove from the fabrication area. On the contrary, the virtual image is easier to remove from the fabrication area than the real image when the hologram is set after the objective lens. In this condition, however, the position of the hologram is restricted by the working distance of the objective lens, and the diffraction efficiency also limits the position of the hologram because the diameter of the pulse becomes small rapidly as the distance increases between the objective lens and the hologram. In view of these facts, we used the virtual image for fabrication.

By considering the results in Figs. 3 and 4, the focal length of the hologram should be short, and so should be the distance between the hologram and the objective lens. Although the diffractive efficiency of the hologram decreases if the focal length of the hologram becomes shorter, the short distance between the hologram and the objective lens compensates for the loss.

\section{3D microfabrication}

By controlling the light intensity profile and the focal length of the hologram as discussed above, we successfully fabricated 24 dots arrayed as a spiral inside silica glass (see Fig. 5). The left side shows the model images and the right side shows optical micrographs of the top and side views of the fabrication (upper and lower images, respectively). The results of our previous paper ${ }^{19}$ are also shown for comparison. The dots in our latest work have consistent spherical shape and have no elongation along the optical axis. In this paper, we used only phase holograms. However, it is well known that not only the phase image but also the amplitude image should be used for better reconstruction image. ${ }^{32}$ It means that our result has growth potential by using phaseamplitude hologram.

This 3D microfabrication technique can fabricate not only dots but also continuous structures. Our group has recently fabricated $1 \mathrm{~cm}$ long waveguides by this technique (only by one shot and without any stage control) and verified the propagation loss as $<1.0 \mathrm{~dB} / \mathrm{cm}$ at a $1550 \mathrm{~nm}$ wavelength. ${ }^{33}$ Although the line structure is fabricated in the plane perpendicular to the optical axis, this technique allows for the line structure fabrication inside this plane if the elongation-free fabrication is achieved. Figure 6 shows a micrograph of a V-shaped line microstructure fabricated inside silica glass as well as a model 3D image. This
V-shaped line spreads out in the plane parallel to the optical axis, which is the first result of its kind as far as we know.

\section{SUMMARY}

We developed a holographic 3D microfabrication technique that uses only a single femtosecond laser pulse and a hologram. By using this technique together with light intensity distribution control, multiple dots of homogeneous size can be fabricated simultaneously. Moreover, choosing an adequate hologram focal length makes it possible to fabricate elongation-free dots and lines without the duplication caused by the real and virtual image of the hologram. By combining these techniques, we succeeded in fabricating a homogeneous and elongation-free spiral dot array and V-shaped line microstructures inside silica glass.

\section{ACKNOWLEDGMENT}

This study was carried out in the High-Efficiency Processing Technology for Three-Dimensional Optical Devices Project as part of the Nanotechnology Materials Program supported by the New Energy Industrial Technology Development Organization (NEDO).

${ }^{1}$ K. M. Davis, K. Miura, N. Sugimoto, and K. Hirao, Opt. Lett. 21, 1729 (1996).

${ }^{2}$ J. Suzuki, M. Yamaji, and S. Tanaka, Proc. SPIE 7201, 72011C (2009).

${ }^{3}$ A. M. Streltsov and N. F. Borrelli, Opt. Lett. 26, 42 (2001).

${ }^{4}$ W. Watanabe, T. Asano, K. Yamada, K. Itoh, and J. Nishii, Opt. Lett. 28, 2491 (2003).

${ }^{5}$ K. Kawamura, N. Sarukura, M. Hirano, and H. Hosono, Jpn. J. Appl. Phys., Part 2 39, L767 (2000).

${ }^{6}$ E. N. Glezer, M. Milosavljevic, L. Huang, R. J. Finlay, T.-H. Her, J. P. Callan, and E. Mazur, Opt. Lett. 21, 2023 (1996).

${ }^{7}$ C. Hnatovsky, R. S. Taylor, P. P. Rajeev, E. Simova, V. R. Bhardwaj, D. M. Rayner, and P. B. Corkum, Appl. Phys. Lett. 87, 014104 (2005).

${ }^{8}$ Y. Cheng, K. Sugioka, and K. Midorikawa, Opt. Lett. 29, 2007 (2004).

${ }^{9}$ A. Marcinkevicius, S. Juodkazis, M. Watanabe, M. Miwa, S. Matsuo, H. Misawa, and J. Nishii, Opt. Lett. 26, 277 (2001).

${ }^{10}$ Y. Li, K. Itoh, W. Watanabe, K. Yamada, D. Kuroda, J. Nishii, and Y. Jiang, Opt. Lett. 26, 1912 (2001).

${ }^{11}$ E. N. Glezer, M. Milosavljevic, L. Huang, R. J. Finlay, T.-H.-H. Her, J. P. Callan, and E. Mazur, Opt. Lett. 21, 2023 (1996).

${ }^{12}$ J. H. Strickler and W. W. Webb, Opt. Lett. 16, 1780 (1991).

${ }^{13}$ B. C. Stuart, M. D. Feit, S. Herman, A. M. Rubenchik, B. W. Shore, and M. D. Perry, Phys. Rev. B 53, 1749 (1996).

${ }^{14}$ R. R. Gattass and E. Mazur, Nat. Photonics 2, 219 (2008).

${ }^{15}$ D. M. Krol, J. Non-Cryst. Solids 354, 416 (2008).

${ }^{16}$ K. Itoh, W. Watanabe, S. Notle, and C. B. Schaffer, MRS Bull. 31, 620 (2006).

${ }^{17}$ E. N. Glezer and E. Mazur, Appl. Phys. Lett. 71, 882 (1997).

${ }^{18}$ M. Yamaji, H. Kawashima, J. Suzuki, and S. Tanaka, Appl. Phys. Lett. 93, 041116 (2008).

${ }^{19}$ M. Yamaji, H. Kawashima, J. Suzuki, and S. Tanaka, J. Optoelectron. Adv. Mater. 12, 474 (2010).

${ }^{20}$ Y. Ohtake, N. Fukuchi, N. Matsumoto, H. Ito, and T. Hara, Opt. Lett. 32, 1411 (2007)

${ }^{21}$ M. Sakakura, T. Sawano, Y. Shimotsuma, K. Miura, and K. Hirao, Opt. Exp. 18, 12136 (2010).

${ }^{22}$ N. J. Jenness, K. D. Wulff, M. S. Johannes, M. J. Padgett, D. G. Cole, and R. L. Clark, Opt. Exp. 16, 15942 (2008).

${ }^{23}$ Y. Kuroiwa, N. Takeshima, Y. Narita, S. Tanaka, and K. Hirao, Proc. SPIE 5339, 185 (2004).

${ }^{24}$ Y. Kuroiwa, N. Takeshima, Y. Narita, S. Tanaka, and K. Hirao, Opt. Express 12, 1908 (2004).

${ }^{25}$ S. Hasegawa, Y. Hayasaki, and N. Nishida, Opt. Lett. 31, 1705 (2006).

${ }^{26}$ J. Bengtsson, Appl. Opt. 33, 6879 (1994). 
${ }^{27}$ E. N. Glezer, Ph. D. dissertation (Harvard University 1996).

${ }^{28}$ M. Shimizu, M. Sakakura, M. Ohnishi, Y. Shimotsuma, T. Nakaya, K. Miura, and K. Hirao, J. Appl. Phys. 108, 073533 (2010).

${ }^{29}$ M. Sakakura, T. Tochio, M. Eida, Y. Shimotsuma, S. Kanehira, M. Nishi, K. Miura, and K. Hirao, Opt. Exp. 19, 17780 (2011).
${ }^{30}$ Y. Peng, Y. Wei, and Z. Lv, Opt. Appl. 34, 319 (2004).

${ }^{31}$ E. Hecht and A. Zajac, Optics (Addison-Wesley, 2001).

${ }^{32}$ V. Mizeikis, S. Judkazis, J. Ye, A. Rode, S. Matsuo, and H. Misawa, Thin Solid Films 445, 438-439 (2003).

${ }^{33}$ J. Suzuki, Y. Arima, and S. Tanaka, Proc. SPIE 7925, 79251D (2011). 